\title{
Unilineage monocytopoiesis in hematopoietic progenitor culture: switching cytokine treatment at all Mo developmental stages induces differentiation into dendritic cells
}

\author{
E Montesoro, ${ }^{*, 1}$, G Castelli ${ }^{1}$, O Morsillii ${ }^{1}$, R Nisini ${ }^{2}$, MH Stafsnes ${ }^{1}$, \\ A Carè ${ }^{1}, C$ Peschle ${ }^{1}$ and $C$ Chelucci ${ }^{1}$ \\ ${ }^{1}$ Department of Hematology, Oncology and Molecular Medicine, Istituto \\ Superiore di Sanità, Rome, Italy \\ 2 Department of Infectious, Parasitic and Immune-mediated Diseases, Istituto \\ Superiore di Sanità, Rome, Italy \\ * Corresponding author: E Montesoro, Department of Hematology, Oncology \\ and Molecular Medicine, Istituto Superiore di Sanità, Viale Regina Elena 299, \\ Rome 00161, Italy. Tel: + 390649902088 ; Fax: + 390649387087 ; \\ E-mail: e.montes@iss.it
}

Received 07.3.05; revised 13.6.05; accepted 11.7.05; published online 19.8.05 Edited by G Cossu

\begin{abstract}
We have developed a new culture system whereby human hematopoietic progenitors purified from adult peripheral blood extensively proliferate and gradually differentiate into $>95 \%$ pure monocytic (Mo) cells. At all developmental stages treatment with interleukin (IL)-4 + granulocyte-macrophage colony-stimulating factor or IL-4 + c-Kit-ligand + FLT-3 ligand switched the Mo precursors into dendritic cells (DCs). The switching capacity decreased only at the end of the culture, when most Mo cells matured to macrophages. Moreover, the Mo precursors were highly susceptible to transduction with lentiviral vectors: once switched to DCs, they maintained the transgene expression, as well as the phenotype and function of the $D C$ lineage. Our results provide new insight into the potential role of the Mo lineage as a reservoir of DCs in vivo. Furthermore, the methodology for transduction of Mo precursors provides a tool to generate genetically modified, normally functioning DCs potentially useful for immunotherapy.

Cell Death and Differentiation (2006) 13, 250-259.

doi:10.1038/sj.cdd.4401748; published online 19 August 2005
\end{abstract}

Keywords: cytokines; dendritic cell differentiation

Abbreviations: APC, antigen-presenting-cell; BM, bone marrow; BSA, bovin serum albumin; DC, dendritic cell; FITC, fluorescein isothiocyanate; FL, FLT-3 ligand; GFP, green fluorescence protein; GM-CSF, granulocyte-macrophage colony-stimulating factor; GM-CSFR, GM-CSF receptor; HLA, human leukocyte antigens; HPC, hematopoietic progenitor cell; IFN, interferon; IL, interleukin; KL, c-Kit-ligand; LPS, lipopolysaccharide; MACS, magnetic cell sorter; M-CSF, macrophage colonystimulating factor; MHC, major histocompatibility complex; MFI, mean fluorescence intensity; MLR, mixed leukocyte reaction;
MoAb, monoclonal antibody; Mo, monocytic; Mos, monocytes; NK, natural killer; PB, peripheral blood; PBMCs, PB mononuclear cells; PE, phycoerythrin; TGF, transforming growth factor; TNF, tumor necrosis factor

\section{Introduction}

Dendritic cells (DCs) are sentinels for the immune system. Located in the periphery in an immature form, upon encounter of microbial antigens or proinflammatory cytokines, the DCs mature, process, and present the internalized antigens in combination with major histocompatibility complex (MHC) molecules. Mature DCs then prime naïve T cells, leading to both effector T-cell differentiation and memory T-cell expansion. ${ }^{1-4}$

In humans, DCs represent a heterogeneous population that may arise from different hematopoietic progenitors/precursors along distinct differentiation pathways. Two intermediate progenitors of DCs have been hypothesized: one expressing myeloid cells-related antigens, which gives rise to DC1 and intrathymic DC1-like cells, as well as monocytes (Mos) and granulocytic cells; 5 the other one with lymphoid but not myeloid potential, able to generate thymic and peripheral DC2 in addition to T, B and NK cells. ${ }^{7-9}$ Even if some authors suggest that these intermediate progenitors belong to different lineages, they may represent different stages of maturation of a unique lineage. ${ }^{10-12}$

DCs derive in their ontogenic profile from stem cells committed to a hematopoietic developmental pathway. ${ }^{13-17}$ The complex mechanisms at the basis of hematopoietic progenitor cells (HPCs) differentiation into specific lineages and the subsequent migration of the committed elements from bone marrow (BM) to peripheral districts, have been elucidated by in vitro and in vivo studies on the differentiative potential of intermediate progenitors, and by the phenotypical and functional characterization of the derived mature progeny. ${ }^{18,19}$

In vitro generation of DC populations has been described to occur in several culture systems. DCs can be obtained from HPCs isolated from cord blood, peripheral blood (PB) and $\mathrm{BM},{ }^{14,20-22}$ or directly from $\mathrm{PB}$ mature Mos, ${ }^{23-25}$ using different cytokines, in combination or alone. ${ }^{26-28}$ Granulocyte-macrophage colony-stimulating factor (GM-CSF) + tumor necrosis factor (TNF)- $\alpha$ and GM-CSF + interleukin (IL)-4 were initially used to induce DC differentiation of HPCs and mature Mos, respectively. ${ }^{14,25}$ Although GM-CSF is a relevant factor, at least in theory, its exclusive use in vitro could underestimate the DC differentiative potential of precursors, since some of them do not express the GM-CSF receptor (GM-CSFR). Recent reports ${ }^{29}$ have demonstrated that an opportune mix of other cytokines can support DC differentiation. Among these cytokines, FLT-3 ligand (FL) was particularly interesting. Its ability to induce in vitro proliferation of 
HPCs as well as to support, in conjunction with other specific cytokines, the differentiation of several hematopoietic lineages is well known. ${ }^{30}$ In vivo studies have revealed its capability to enhance the number of circulating DCs both in mice $^{31}$ and in humans. ${ }^{32}$ Furthermore, c-Kit-ligand (KL), known to induce cell proliferation in diverse hematopoietic lineages, has been used in several DC culture systems. ${ }^{33}$

In this study, we describe a liquid culture system that allows unilineage Mo differentiation/maturation from CD34 ${ }^{+}$HPCs. Using two different cytokine combinations, Mo cells were induced, at different days of development, to differentiate into DCs. The results indicate that Mo precursors maintain the bipotential capability to generate not only Mos but also DCs throughout their differentiation/maturation, from early committed precursors to mature Mos. Furthermore, gene transfer experiments showed that Mo precursors and DCs obtained by this culture system are highly susceptible to lentiviral transduction. These studies provide a tool to analyze at cellular and molecular level the developmental relationship between the two lineages and to obtain large numbers of genetically modified DCs, which may be used in novel strategies of immunointervention.

\section{Results}

\section{Mo unilineage culture}

Treatment of highly purified HPCs with optimal macrophage colony-stimulating factor (M-CSF), IL-6 and FL dosages in the presence of FCS (here referred to as 'primary culture') resulted in significant cell proliferation (i.e. 100-400-fold cell number amplification) (Figure 1a), as well as selective Mo differentiation/maturation (Figures $1 \mathrm{~b}$ and $2 \mathrm{a}-\mathrm{d}$ ). In particular, in the first week of culture, HPCs proliferated and concomitantly differentiated towards the Mo lineage, as assessed by the progressive downmodulation of the HPC-associated CD34 antigen (from $\geq 95 \% \mathrm{CD} 4^{+}$cells at day 1 to $\sim 20 \%$ at day 7) and by the appearance of Mo-specific CD14 antigen, present on $\sim 60 \%$ of cells after 7 days of culture. In the very first days of culture (Figure 2a), morphological evaluation indicated that the cell population consisted of about $90 \%$ small blasts, while the remaining cells were mainly monoblasts, characterized by a deep blue cytoplasm and a large, eccentric and centrally indented nucleus with one or two large nucleoli, and rare promonocytes, that is, larger cells with a lobated nucleus and a clear blue cytoplasm containing few azzurrophilic granules. Noteworthily, the relative percentage of these cell types markedly changed from days 1 to 7 of culture, in correlation with the differentiative stage. In the second week, CD34 antigen expression was almost completely lost (being present on less than $4 \%$ of cells at day 14) whereas, at the same time, a gradual increase of $\mathrm{CD}_{14}{ }^{+}$cells could be observed. Early in the second week (Figure $2 b$ ), the culture approximately consisted of $15 \%$ blasts, $60 \%$ monoblasts, $15 \%$ promonocytes and 5\% Mos. At days 13-15 (Figure 2c), cells were represented by about $5 \%$ monoblasts, $10 \%$ promonocytes, $65 \%$ Mos, recognizable by a reniform nucleus that occupies a half of the cell volume and by a finely granulated, gray blue cytoplasm, and about 15\% macrophages, larger cells with an eccentric small nucleus and a a

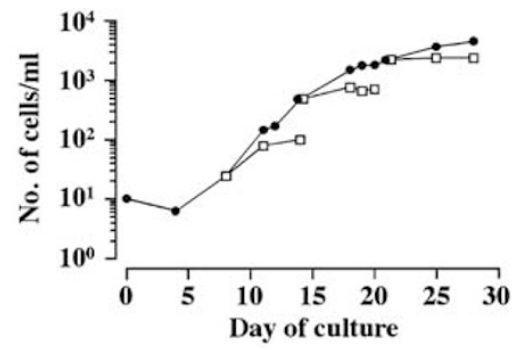

b
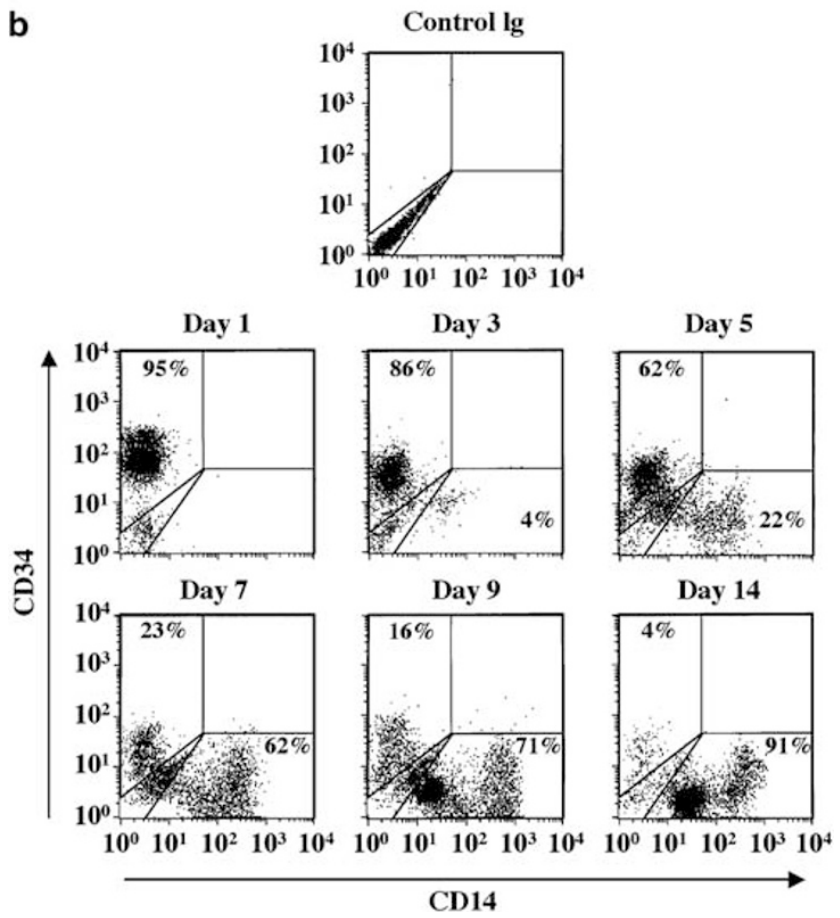

Figure 1 Characterization of unilineage Mo culture. (a) Absolute numbers of total viable cells during Mo unilineage culture $(\mathbf{O})$. Mo precursors were switched to DCs at different days of culture $(8,14$ and 21 in this experiment) $(\square)$. Results are representative of seven independent experiments. (b) Kinetics of CD34 and CD14 expression on HPCs cultivated with FL, IL-6 and M-CSF. At the indicated time points, aliquots of cells were collected and double stained with anti-CD14FITC and anti-CD34-PE MoAbs. Quadrants were set according to the fluorescence intensities of FITC- and PE-conjugated isotype-matched control IgGs. Results are representative of three experiments

cytoplasm highly vacuolated. In the third week, the developed progeny resulted in $95-100 \% \mathrm{CD}^{+} 4^{+}$cells, consisting mainly of mature Mos, and about 30\% macrophages. Extending the time of culture up to 25-30 days (Figure 2d), the proliferation rate became consistently slower than before and the terminal differentiation of Mos to macrophages was almost complete. Indeed, at this time of culture, roughly a half of the cells expressed the CD86 antigen (data not shown). A more detailed phenotypical characterization was hampered by the absence of monoclonal antibodies (MoAbs) able to discriminate human Mo cells at the different maturative stages. In fact, CD31 was expressed on virtually all the cells during the entire culture period, while CD11b showed an upmodulation with kinetics that was very similar to that of CD14, but still not specific for the cells at the differentiative stages preceding that of mature Mos (data not shown). 

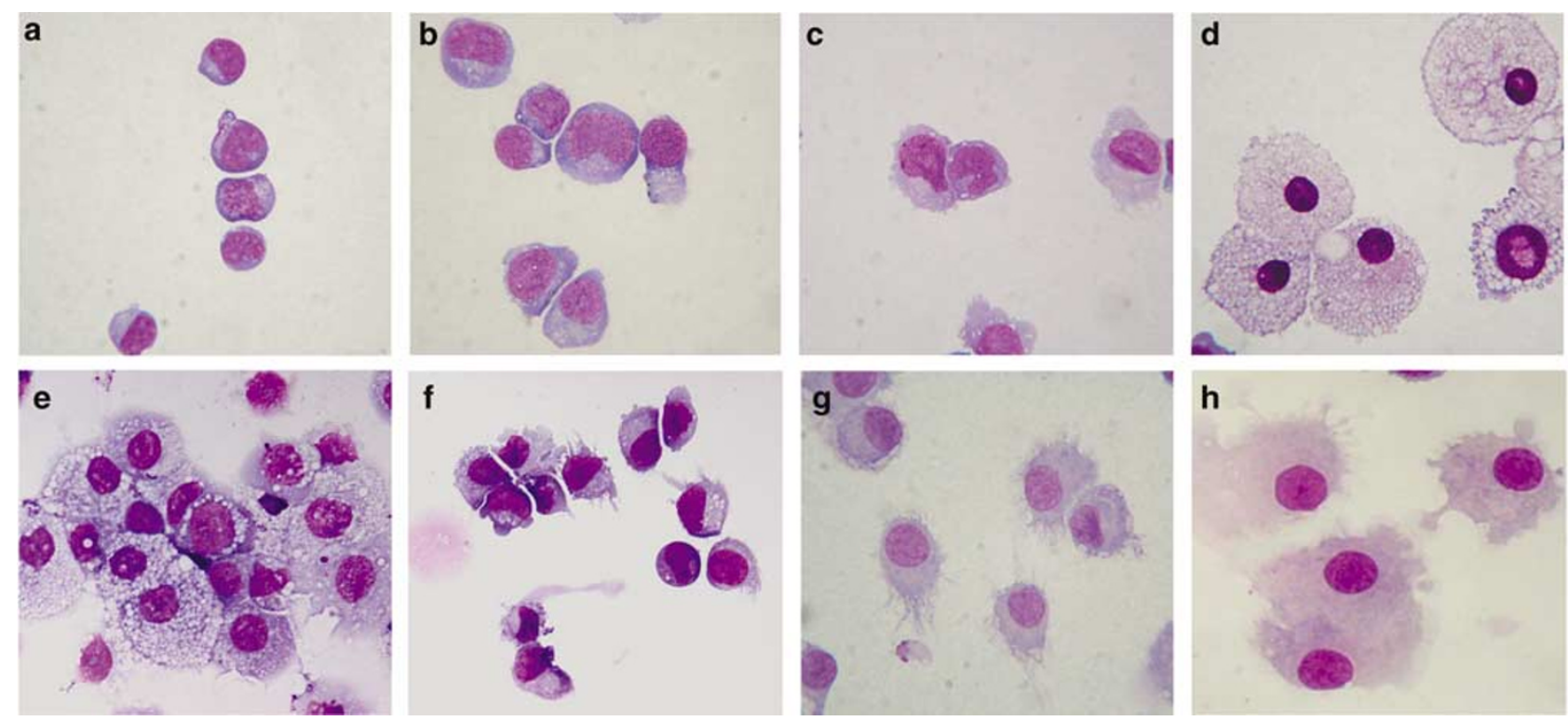

Figure 2 Morphology of cells from Mo culture and their derived DC progeny. Mo populations at day 2 (a), day 8 (b) and day 14 (c) of the primary culture. Typical macrophagic features appear after 25-30 days of culture in Mo medium (d). CD14 $4^{\text {bright }}$ cells (e) and CD14 ${ }^{\text {dim }}$ cells (f) sorted at day 14 of the Mo culture. DC populations, derived from day 14 Mo precursors after 5 day treatment with IL-4 + GM-CSF and IL-4 + FL + KL, respectively, and terminally activated by adding LPS (e and f) (original magnification $\times 400$ )

In the majority of the experiments, we could detect CD14 dim and CD14 ${ }^{\text {bright }}$ cells at different times of the Mo culture (see Figure 1b). Forward side scatter and morphological analysis indicated that they represent cell subsets at different maturative stages. In particular, almost all the CD14 ${ }^{\text {bright }}$ population consisted of more mature cells with premacrophagic features, that is abundant and highly vacuolated cytoplasm (Figure 2e), while the majority of the day 14-sorted CD14 ${ }^{\text {dim }}$ cells displayed a reniform nucleus and a pale and finely granulated cytoplasm, peculiar of Mo cells (Figure 2f).

Analysis of GM-CSFR expression revealed that this receptor was already detectable on Mo precursors at days 4-5 of culture and continued to be present during all the differentiative/maturative stages. Later on, about $70 \%$ of the total cells were GM-CSFR ${ }^{\text {hi }}$ (see below).

Several DC-specific membrane antigens, such as CD40, CD80, CD83 were never detected on Mo precursors and mature Mos, while the expression of CD1a never exceeded $10 \%$ of the cell number. Finally, cell staining with CD15 MoAb during the entire culture period excluded the presence of basophils (data not shown).

Occasionally, at day 0 we observed $\sim 1 \% \mathrm{CD} 34^{+} 14^{+}$cells together with $\geq 95 \%$ CD34 ${ }^{+} 14^{-}$cells (results not shown). To assess the relative contribution of $\mathrm{CD} 34^{+} 14^{-}$and $\mathrm{CD} 34^{+} 14^{+}$cells to generate the unilineage population described above, each cell type was sorted and utilized as a starting population for the Mo culture (and then switched to DC culture conditions, see below). The results obtained using $\mathrm{CD} 34^{+}$or $\mathrm{CD} 34^{+} 14^{-}$progenitors as starting population were overlapping, in terms of both proliferative and differentiative capabilities, whereas the CD34 ${ }^{+} 14^{+}$subset did not show any growth potential in the presence of an Mo-specific cytokine cocktail and died within a few days (data not shown).

\section{Generation of DCs from Mo precursors}

To identify at which differentiative stage(s) Mo cells could acquire the capacity to switch toward the DC lineage, Mo precursors between days 7 and 21 of culture were treated with either IL-4 + GM-CSF or IL-4 + KL + FL for 5 days. Samples of the cells were further treated overnight with lipopolysaccharide (LPS) to verify the capacity of acquiring the functional and phenotypical characteristics of mature DCs. The differentiation to DCs, irrespective of the culture medium used, was consistently coupled with a reduced proliferation rate with respect to Mo precursor growth (Figure 1a). This effect was particularly evident at the late phases of the primary culture. Treatment of Mo cells with both DC media gave rise to DCs, and the culture period required for the generation of typical DCs was dependent on the differentiative stage of the primary culture. In summary, days 7-10 Mo precursors required 10-13 days to differentiate into DCs, while days 13-21 Mo cells required a culture of 5-6 days. Results reported below refer mainly to Mo precursors induced at days 13-15.

Morphological modifications started very early, that is $2-3$ days after resuspending the cells in the DC specific media; after additional 3 days of culture cells acquired the distinct DC morphology, which differed in the two culture conditions. Namely, the DCs obtained after IL-4 + GM-CSF treatment were characterized by a monocytoid nucleus and an abundant, pale basophilic cytoplasm, endowed with a minor azzurophilic granulation, irregular margins and fine cell processes, often polarized (Figure 2g); whereas the DCs obtained after incubation with IL-4 + KL + FL were larger, with a more abundant and pale cytoplasm and margins shaped as lamellipodia or veils (Figure 2h).

For a better characterization of the two DC populations, extensive phenotype analyses were conducted at the end of 
culture, before and after LPS stimulation. Figure 3 demonstrates a representative experiment, where Mo precursors at day 14 of culture were induced to differentiate into DCs by IL$4+$ GM-CSF (Figure 3a) or IL-4 + KL + FL (Figure 3b) treatment. The data indicate that CD14 antigen was significantly, if not completely, downmodulated and that the mean fluorescence intensity (MFI) of CD14 ${ }^{+}$DCs was always lower than that of $\mathrm{CD} 14^{+}$Mo cells, being about 30 and 70 , respectively

a
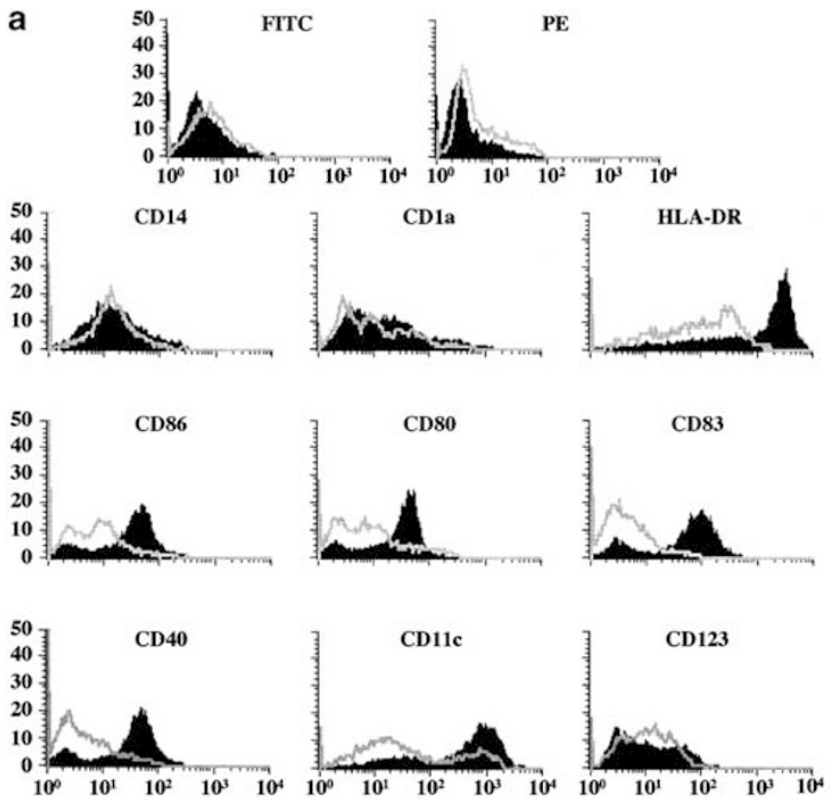

b
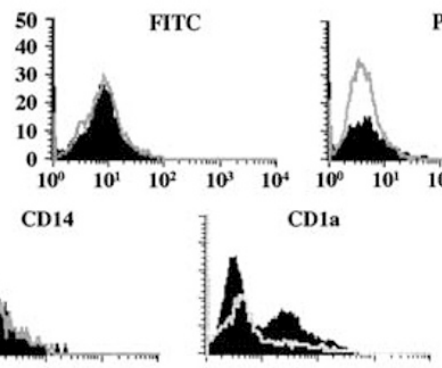

PE
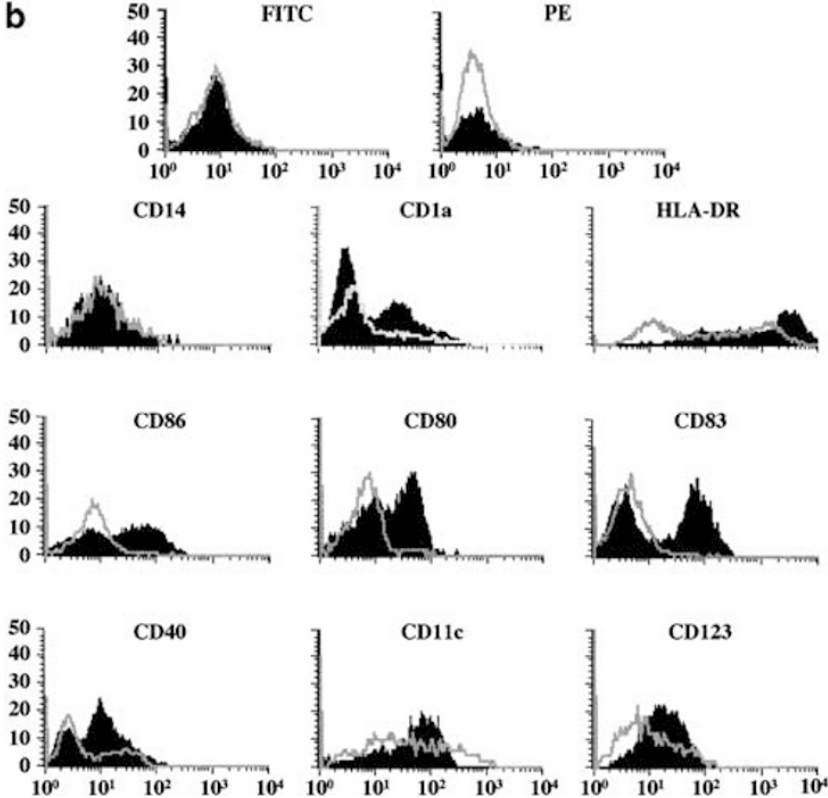

Figure 3 Phenotypic profiles of DCs derived from Mo precursors. DCs were obtained from Mo precursors treated with GM-CSF and IL-4 (a) or KL + FL + IL4 (b). Cells were analyzed before (gray line) and after (filled plot) $16 \mathrm{~h}$ LPS stimulation, by flow cytometry, for surface antigens expression. The following FITC-conjugated MoAbs: CD14, CD1a, HLA-DR, CD86, CD80, and PEconjugated MoAbs: CD83, CD40, CD11c, CD123, were used. Appropriate conjugated isotype-matched IgGs were used as control. Data from one representative experiment out of five are reported (mean from four experiments). Moreover, the retention of CD14 was more evident when the switching of Mos to DCs was performed later during the culture period (days 17-21). DCs obtained upon IL-4+GM-CSF culture showed an increase up to $30-65 \%$ of the CD1a marker. After LPS stimulation, an upregulation of DC markers (i.e. CD80, CD40, CD83, CD86 and human leukocyte antigens (HLA)-DR) was observed. The phenotypic profile of the DCs derived from Mo precursors treated with IL-4 $+\mathrm{KL}+\mathrm{FL}$ was similar, with the exception of CD83 and CD40 that were expressed by a lower percentage of cells (i.e. $26 \% \pm 8$ and $20 \% \pm 6$ versus $60 \% \pm 15$ and $55 \% \pm 14$, respectively).

The CD11c antigen, variably expressed on the starting Mo population, was virtually present on all the derived DCs, whereas CD123 marker, namely the $\alpha$ chain of IL-3 receptor, highly expressed on plasmacytoid DCs, was present even if at low intensity of fluorescence on $30-40 \%$ of cells. The apparent discrepancy between this latter result and the myeloid origin of the DCs was further investigated. We found that, at odds with plasmacytoid control DCs isolated from $\mathrm{PB}$ mononuclear cells (PBMCs), our DC cultures were BDCA-2 and BDCA-4 negative and unable to undergo phenotypical maturation and interferon (IFN)- $\alpha$ secretion upon CpG stimulation (data not shown). The negative results in these assays were consistent with the myeloid origin of DCs and excluded a plasmacytoid DC differentiation from CD34derived Mo precursors.

Interestingly, the immunofluorescence analyses indicated that the capability of Mos to differentiate into DCs was completely lost at the end of the culture (days 25-30) when the majority of cells were adherent macrophage-like cells. To confirm this observation, the adherent and nonadherent cells of the primary culture were separated at day 20 and induced into DCs by cultivating them with IL-4 + GM-CSF for 6 days. The cell population derived from the adherent fraction was about $85 \% \mathrm{CD}^{+} 4^{+}$and less than $1 \% \mathrm{CD} \mathrm{C}^{+}$. On the contrary, DCs derived from the nonadherent fraction were 25 and $55 \% \mathrm{CD} 14^{+}$and CD1a ${ }^{+}$, respectively (Figure 4). The same results were obtained by cultivating the two populations in the presence of IL-4 $+\mathrm{KL}+\mathrm{FL}$ (data not shown).

\section{Correlation between the expression of GM-CSFR and DC induction by IL4 plus GM-CSF treatment}

Since we observed that early Mo precursors (days 7-10) needed a longer culture period to switch to DCs, as compared to late Mo cells (days 13-21), we tested a hypothetical correlation of this finding with the GM-CSFR expression on the cell surface. As described above, the GM-CSFR appeared very early in Mo cultures and both GM-CSFR ${ }^{\text {hi }}$ and GMCSFR $^{\text {lo }}$ cell populations were under constant observation from days 7 to 14 . Double staining of day 14 cells with antiCD86 (highly expressed on macrophages) and anti-GMCSFR MoAbs detected the presence of $\mathrm{CD}^{-} 6^{-} \mathrm{GM}^{-C S F R}{ }^{-}$, $\mathrm{CD}^{-} 6^{-} \mathrm{GM}^{-C S F R}{ }^{+}$and CD86 ${ }^{+} \mathrm{GM}-\mathrm{CSFR}^{+}$cell populations, which represented 26,47 and $27 \%$, respectively, of the total cell number (Figure 5, left panel). These fractions were sorted, analyzed by cell morphology and then incubated with IL$4+$ GM-CSF. Giemsa staining revealed the following morphological composition: $15 \%$ monoblasts, $60 \%$ promonocytes 
and $25 \%$ Mos in CD86 ${ }^{-}$GM-CSFR $^{-}$fraction; $15 \%$ promonocytes, $75 \%$ Mos and 10\% macrophages in CD86 ${ }^{-}$GM$\mathrm{CSFR}^{+}$population; $10 \%$ Mos and $90 \%$ macrophages in $\mathrm{CD}^{+}{ }^{+} \mathrm{GM}-\mathrm{CSFR}^{+}$subset. After 5 days of IL-4 + GM-CSF treatment, virtually all the cells derived from the $\mathrm{CD}^{-} 6^{-} \mathrm{GM}$ $\mathrm{CSFR}^{+}$fraction were CD1a ${ }^{+} \mathrm{CD} 14^{-}$, whereas the majority of those derived from the $\mathrm{CD}^{+} 6^{+} \mathrm{GM}^{-C S F R}{ }^{+}$fraction were $\mathrm{CD}_{14}{ }^{+}$, with a low percentage of CD1a ${ }^{+}$cells. The CD86 ${ }^{-}$ GM-CSFR $^{-}$population consisted of more immature cells, in fact at least 13-15 days of IL-4 + GM-CSF treatment were needed for the appearance of DCs, as measured by the expression of CD1a marker, which reached $50 \%$ of positive cells (Figure 5, right panel). Unfortunately, it was not possible to further examine the CD1a marker expression of this cell population because after 25-30 days of culture (i.e. 14 days for in vitro differentiation in Mo conditions plus 2 weeks as DC culture) cells began to die.

\section{Functional activities of DCs derived from Mo precursors}

Antigen uptake is a typical immature DC activity. DCs derived from Mo precursors, induced to differentiate either with
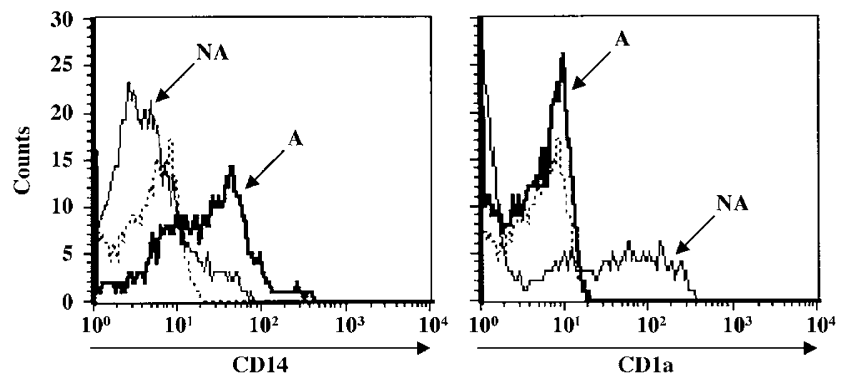

Figure 4 Phenotypic analysis of DCs derived from adherent (a) and not adherent (NA) cell fractions of Mo precursors. At day 20 of primary culture, the nonadherent cell fraction was separated from the adherent one by removing the supernatant. After two washings with PBS, the remaining adherent cells were detached by gentle scraping. Then, the two cell fractions were induced to DC differentiation with GM-CSF + IL-4 and, after a 5-day culture, examined for expression of CD14 (left panel) and CD1a (right panel) antigens. Hatched line indicates the isotypic control. A representative experiment is shown
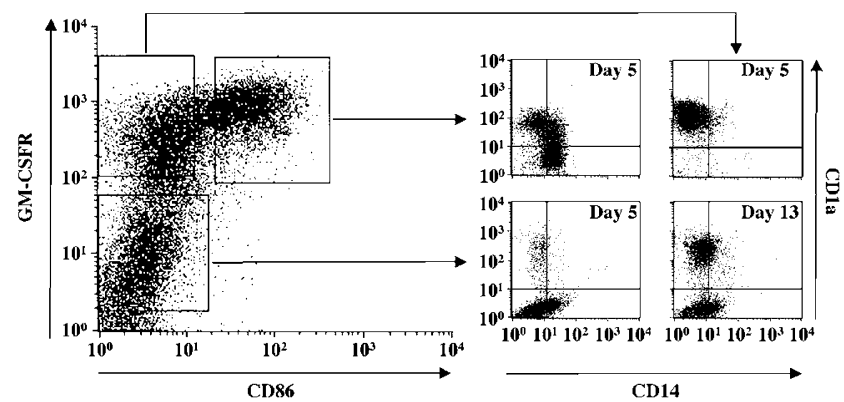

Figure $5 \mathrm{DC}$ differentiative potential of Mo cells. Identification of three distinct subsets of Mo precursors at day 14 of culture, based on GM-CSFR and CD86 expression (left panel). Sorted cells were seeded in the presence of GMCSF + IL-4 and after 5 days analyzed for CD1a and CD14 expression by double color fluorescence (right panel). The phenotypic profile of sorted GM$\mathrm{CSFR}^{-} \mathrm{CD}^{-} 6^{-}$cells was repeated at day 13 of culture
$\mathrm{IL}-4+\mathrm{GM}-\mathrm{CSF}$ or with IL-4 + KL + FL, were tested for their macropinocytosic capability by measuring the uptake of fluorescein isothiocyanate (FITC)-conjugated dextran. Flow cytometry analysis showed that $45-90 \%$ of immature DCs efficiently captured FITC-dextran. DCs derived from PB Mos were also tested as positive control (Figure 6a).

The most distinctive feature of mature DCs is their ability to strongly stimulate the activation of allogenic $T$ cells. This functional activity was assessed by the mixed leukocyte reaction (MLR) assay, which revealed a comparable stimulatory capability of DCs derived from Mo precursors treated with both GF combinations. Interestingly their activity was consistently higher than that of PB mature Mos, but slightly weaker with respect to DCs derived from PB mature Mos, included as a reference population (Figure $6 \mathrm{~b}$ ). The latter results are explained by the asynchronism of the Mo precursor cultures giving rise to the DC populations. In fact, as shown in Figures $3 a$ and $b$, not all the Mo precursors undergo differentiation into the dendritic lineage at the same time, therefore the real number of professional antigen-presentingcells (APCs) is lower than that of the reference population.
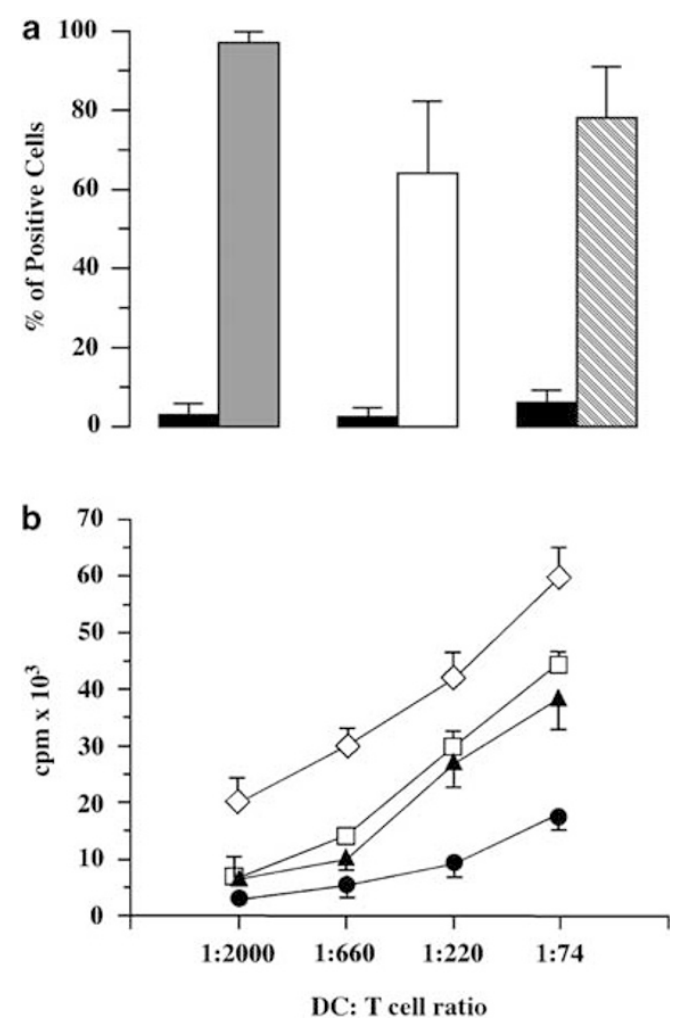

Figure 6 DCs derived from Mo precursors are functionally active. (a) FITCdextran uptake by DCs derived either from PB Mos $(\square)$ or from Mo precursors $(\square)$, both cultivated with GM-CSF and IL-4, and by DCs derived from Mo precursors and cultivated with $\mathrm{IL}-4+\mathrm{KL}+\mathrm{FL}(\mathbb{S})$. As control, identical cell samples were incubated with FITC-dextran at $4^{\circ} \mathrm{C}(\mathbf{\square})$. (b) Induction of allogenic T-cell activation by Mo precursors $(\mathbf{)})$, DCs derived from Mo precursors and incubated 6 days either with GM-CSF plus IL-4 $(\square)$, or with the GF cocktail $(\boldsymbol{\Delta})$ and PB-derived DCs $(\diamond) .{ }^{3} \mathrm{H}$-thymidine incorporation is expressed as the mean of quadruplicates \pm S.D. The amount of ${ }^{3} \mathrm{H}$-thymidine incorporated by $\mathrm{T}$ cells and DCs cultured in the absence of stimulator and responder cells, respectively, were assumed as background values 


\section{Transgene expression in Mo precursors, PB Mos and DCs}

To evaluate the susceptibility to lentiviral transduction of Mo cells and DCs and to establish optimal conditions for generation of gene-modified cells, Mo precursors were infected at days 10-14 of culture and successively grown either in Mo medium or in the presence of IL-4 + GM-CSF. Alternatively, they were first induced towards the DC lineage, by a 5-day treatment with IL-4 plus GM-CSF, and then infected. A similar schedule of infection was followed for mature Mos, as they were either infected soon after their isolation from $\mathrm{PB}$, and then cultured in Mo or in $\mathrm{DC}$ condition, or were first induced to differentiate into DCs and then infected. At 3-10 days after two rounds of transduction, cells were analyzed for green fluorescence protein (GFP) and lineage markers expression. The overall transduction efficiency of the cell cultures derived from PB Mos was very low (GFP-positive cells ranged from 17 to $26 \%$ ), particularly in terms of MFI (ranging from 23 to 33). On the contrary, cell populations derived from Mo precursors were all transduced at significant levels, being GFP positive: $35 \pm 18 \%$ of Mo precursors, $40 \pm 23 \%$ of DCs derived from transduced Mo precursors and $53 \pm 20 \%$ of transduced DCs. It was also constantly observed that the GFP-positive cells derived from Mo precursors showed a MFI considerably higher than that of the analogue cell populations derived from PB Mos, and that directly transduced DCs displayed a MFI slightly lower than that of DCs derived from transduced Mo precursors. In Figure $7 \mathrm{a}$ the product between the percentage of GFPpositive cells and the corresponding MFI value is reported. The lower transduction efficiency observed in some experiments was found to be correlated with the lower titer of the lentiviral vector preparations used. Phenotypical analysis demonstrated that gene transduction did not impair the expression of typical DC markers, since CD1a, CD83 and CD86 were present on about 35,50 and $60 \%$, respectively, of both transduced and untransduced cells (Figure 7b). In addition, antigen uptake and APC activities were both comparable to those displayed by the untransduced DCs (data not shown and Figure 7c).

\section{Discussion}

The present study provides evidence that DCs are generated from Mo cells at any stage of their differentiation/maturation pathway. Our results provide novel insight into the development of DCs, showing that both Mo progenitors/precursors and circulating Mos can directly differentiate into DCs.

First, we characterized the optimal culture conditions to generate a progeny of virtually pure Mos from highly purified $\mathrm{CD} 4^{+}$cells. In a preliminary report ${ }^{34}$ we described the synergistic effect of FL and M-CSF on HPCs to stimulate their Mo differentiation, coupled with a moderate proliferation, resulting in 28 -fold amplification of the initial cell number. In this study, we show that addition of IL-6, known to induce proliferation of myeloma cells and differentiation of macrophages in several myeloma cell lines, ${ }^{35-37}$ strongly improved Mo cell proliferation up to 400 -fold amplification. Moreover, this culture system allowed the maturation of Mos into a

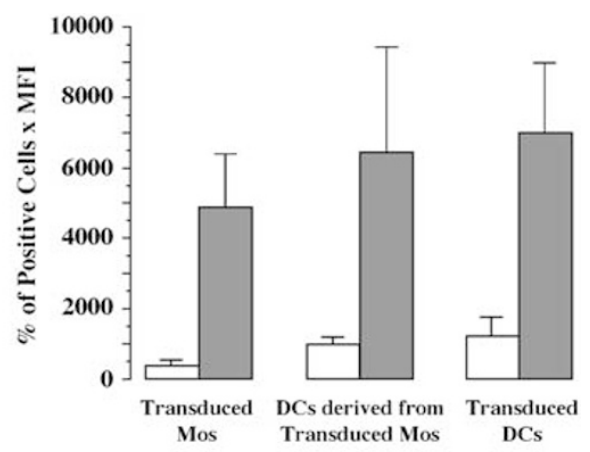

b
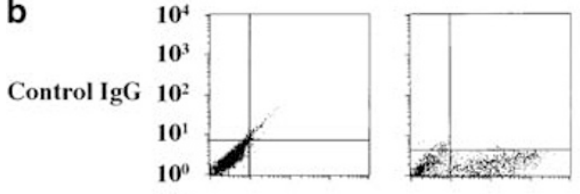

CD1a
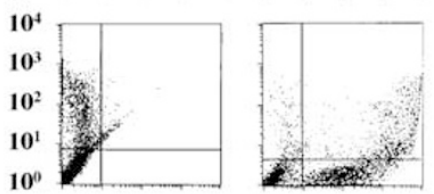

CD83
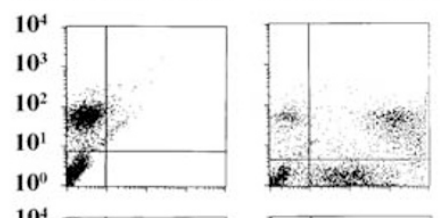

CD86

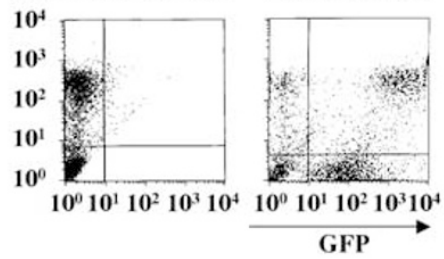

c

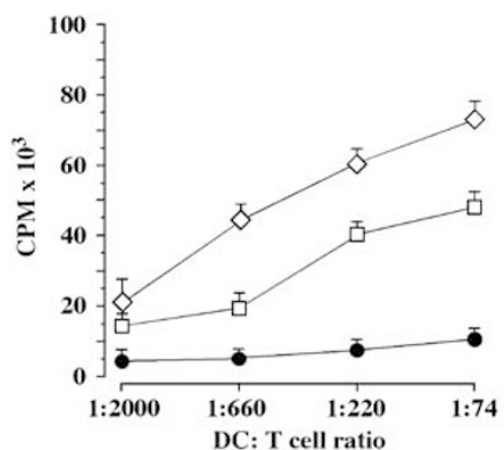

Figure 7 Transduction of Mo and DC cultures. (a) Flow cytometry analysis of transduced Mos, DCs derived from transduced Mos and transduced DCs, all obtained from PB $(\square)$ or Mo precursors $(\square)$. Values of the histogram represent the product between the percentage of GFP-positive cells and the corresponding MFI. The mean of six experiments \pm S.D. is shown. (b) Phenotypic profiles of DCs derived from untransduced (left panels) and transduced (right panels) Mo precursors seeded in GM-CSF and IL-4. After $16 \mathrm{~h}$ LPS stimulation, DCs were analyzed by flow cytometry for the surface antigen expression of CD83, CD86 and CD1a. Appropriate conjugated isotype-matched IgGs were used as control. Data from one representative experiment out of two are reported. (c) Induction of allogenic T-cell activation by transduced Mo precursors $(\bullet)$ and DCs derived from either transduced Mo precursors $(\square)$ or PB Mos $(\diamond) .{ }^{3} \mathrm{H}$-thymidine incorporation is expressed as the mean of quadruplicates \pm S.D. Results are representative of one experiment out of two 
macrophages, as assessed by morphological and phenotypical analyses. Thus, the methodology described here provides a homogeneous and large cell population pertaining to the Mo-macrophage lineage, which allows dissection of the molecular and cellular mechanisms underlying monocytopoiesis. The differentiative stages of Mo lineage were characterized by a gradual decrease of CD34 and a progressive increase of CD14 antigen expression. Noteworthy, the membrane expression of these two molecules was mutually exclusive, as we never observed a CD34 ${ }^{+} 14^{+}$cell population in the differentiating cultures. A CD34 ${ }^{+} 14^{+}$circulating cell population, able to differentiate into DCs when cultured in the presence of GM-CSF, has been described before: ${ }^{38}$ it was hypothesized to represent an intermediate stage of differentiation between HPCs and mature Mos or DCs. Apparently, our Mo culture conditions hamper the growth of doublepositive $\mathrm{CD} 34^{+} 14^{+}$cells; further studies may allow the identification of the GF/cytokine cocktail permissive for the in vitro production of this cell population, thus allowing the study of its role in both DC and Mo differentiative pathways.

Second, we induced Mo cells at various stages of differentiation to switch into DCs by using IL-4 + GM-CSF or $\mathrm{IL}-4+\mathrm{KL}+\mathrm{FL}$. After 5 days, cells acquired all morphological, phenotypical and functional features of DCs. They expressed all typical DC markers and, as expected, CD11c was present on virtually all DCs. On the other hand, $30-40 \%$ of cells expressed low levels of CD123, a marker that defines plasmacytoid DCs, ${ }^{39,40}$ but were BDCA-2 and BDCA-4 negative and unable to undergo phenotypical maturation and IFN- $\alpha$ secretion upon $\mathrm{CpG}$ stimulation. These results are consistent with the myeloid origin of DCs and exclude the plasmacytoid DC differentiation from HPC-derived Mo precursors. Mo precursors-derived immature DCs were able to internalize soluble antigens and, once completely mature, displayed a strong APC activity in allogeneic MLR assay. Noteworthy, we constantly detected $<10 \%$ of $\mathrm{CD} \mathrm{a}^{+}$cells in Mo cultures. It seems unlikely that these few $\mathrm{CD} \mathrm{a}^{+}$cells represent a major source of DCs in the cultures: indeed, after a 5-day treatment with $\mathrm{IL}-4+\mathrm{GM}-\mathrm{CSF}$ or $\mathrm{IL}-4+\mathrm{KL}+\mathrm{FL}$ approximately 60 or $30 \%$ of the cells, respectively, expressed this marker, in spite of a very low cell proliferation rate. The bipotential capability of Mo precursors to generate both Mos/ macrophages and DCs was maintained until approximately 20-25 days; thereafter, Mos undergo maturation into macrophages and their capability to differentiate into DCs is almost completely lost.

Although a wide variety of cytokine combinations have been described to induce mature Mos or HPCs to switch into DCs, the majority of reports indicate GM-CSF and IL-4 as essential factors for DC differentiation. Other cytokines shown to play a role in generating DCs from several precursors, under various culture conditions, include IL-2, IL-3, IL-6, IL-7, IL-12, IL-13, CD40 ligand, FL and transforming growth factor (TGF) $\beta$, alone or in combination. ${ }^{29}$ In particular, FL has a significant effect on DC development both in vivo and in vitro. Maraskovsky et al. ${ }^{32}$ demonstrated that administration of FL to healthy volunteers was followed by a significant increase of circulating DCs, still very efficient as APCs.Furthermore, Strobl et al. ${ }^{41}$ reported the cooperation between $\mathrm{FL}$ and TGF $\beta 1$ in potentiating the development and the cluster formation of DCs. Here we used FL together with $\mathrm{KL}$, which enhances cell proliferation in several hematopoietic cell lineages $^{42}$ and also promotes DC replication in vivo.

Our findings further show that the capability of Mo precursors to generate DCs after treatment with IL-4 + GMCSF is correlated with the elevated GM-CSFR expression on the cell surface. From days 7 to 14 of Mo culture, we observed the presence of GM-CSFR ${ }^{\text {lo }}$ and GM-CSFR ${ }^{\text {hi }}$ cells. Sorting of these cell populations, and their subsequent treatment with IL-4 + GM-CSF, demonstrated a higher susceptibility of GM-CSFR $^{\text {hi }}$ cells to differentiate into DCs, as compared to GM-CSFR $^{\text {lo }}$ cells, which require a longer culture time. It should be pointed out that the differentiation/maturation of Mo cells was not totally synchronous, as observed by the gradual modulation of all the analyzed membrane markers. This feature may explain why not all Mo precursors simultaneously differentiate into DCs. At late culture stages, cells acquired the morphological and phenotypical properties of macrophages and lost their differentiative potential towards the DC lineage. This observation was confirmed by sorting the cells expressing the CD86 macrophage marker, or by isolating the adherent cell fraction appearing late in the culture. Upon DC induction with $\mathrm{IL}-4+\mathrm{GM}-\mathrm{CSF}$, both the sorted $\mathrm{GM}-\mathrm{CSF}^{+} \mathrm{CD}^{+}{ }^{+}$and the adherent cells did not differentiate into DCs: conversely, they maintained a high CD14 antigen expression and showed the typical morphology of macrophages.

Caux et al. ${ }^{14}$ demonstrated that CD14 ${ }^{+}$precursors deriving from $\mathrm{CD}_{34}{ }^{+}$cells possess a bipotential Mo/macrophagic and DC differentiative capability, when selectively induced. Our studies, while in line with these observations, provide novel methodology and insight into DC development. The culture conditions described by Caux et al. ${ }^{14}$ and our group are basically different, in terms of cytokine treatment and, mainly differ in the pathway to DC differentiation. We induced HPCs to differentiate first along the Mo lineage and then into DCs by using two different cytokine cocktails, whereas they committed the CD34 ${ }^{+}$cells directly towards the DC pathway by adding $\mathrm{KL}+\mathrm{GM}-\mathrm{CSF}+\mathrm{TNF} \alpha$. More important, the CD14 ${ }^{+}$precursors described by Caux et al. differ from those described in this study, in that they lack proliferative capacity, whereas in our culture conditions acquisition of the CD14 antigen was not associated with a decrease in the proliferation rate.

On the basis of our results, we suggest that immature Mos may represent a reservoir of DC precursors that, upon specific stimulation, generate functional DCs. In addition to the in vitro data mentioned above, studies in mice demonstrated that circulating Mos may differentiate into DCs. ${ }^{43}$ On the other hand, surgical stress induces a rapid increase of the number of circulating DCs but not of mature Mo cells, thus indicating that the number of circulating DCs and Mos may be differentially regulated. ${ }^{44}$ This rise of DCs was hypothetically explained by the existence of preformed DCs, marginated in 'storage' sites, that could be rapidly mobilized to prepare the host's immune defense against trauma. ${ }^{44}$ Our results additionally, or alternatively, suggest that Mo precursors, stored in the BM, may represent a remarkable DC source, more rapidly available than HPCs.

We have also established a new methodology to efficiently generate transduced DCs by a two-step culture method: first, 
expansion of Mo precursors from HPCs; second, efficient gene transduction of Mo precursors using lentiviral vector, followed by their differentiation into DCs. This methodology differs from those previously described for transgene expression in DCs, ${ }^{45-47}$ and provides the basis for gene transfer studies on Mo precursors to investigate the functional role of specific genes involved in the regulation of Mo and/or DC differentiation.

\section{Materials and Methods}

\section{HPC, PB monocyte and plasmacytoid DC isolation}

HPCs were purified from PB of healthy donors as previously described. ${ }^{48}$ After a density gradient centrifugation on Lympholyte- $\mathrm{H}$ (Cedarlane, Hornby, Ont., Canada), PBMCs were harvested and CD34 ${ }^{+}$cells were purified using the direct CD34 ${ }^{+}$magnetic cell sorter (MACS) isolation kit (Miltenyi Biotec, Bergisch Gladbach, Germany). The cell population obtained consisted mainly of blasts ( $>95 \%$ ) as evaluated by clonogenic assay $^{49}$ and morphological analysis. In some experiments, the CD34 ${ }^{+}$ population was further sorted by FACSVantageSE or FACSDIVA (BectonDickinson) yielding $>99 \%$ pure $\mathrm{CD} 34^{+}$cells, utilizing anti-CD34 (clone 563) MoAb. Mature Mos were isolated from PB of healthy adult donors Mononuclear cells were first fractionated on Lympholyte-H gradient and then isolated with $\mathrm{CD} 14^{+}$Microbeads kit (MACS of human leukocytes, Miltenyi Biotec). The resulting population consisted of more than $96 \%$ Mos, as confirmed by May-Grünwald Giemsa (Sigma-Aldrich, Steinheim, Germany) staining, forward and side scatter in flow cytometry and immunophenotype. Plasmacytoid DCs were isolated from PB mononuclear cells with the BDCA-4 Cell Isolation Kit, Human (Miltenyi Biotec).

\section{Mo unilineage culture}

Mo unilineage cultures were obtained by cultivating $2 \times 10^{5} \mathrm{HPCs} / \mathrm{ml}$ in the medium previously described ${ }^{49}$ supplemented with $20 \%$ heat-

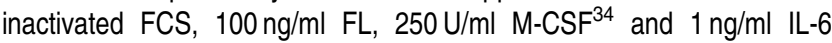
(Peprotech, Rocky Hill, NJ, USA). The cells were cultivated in a fully humidified atmosphere containing $5 \% \mathrm{CO}_{2}, 5 \% \mathrm{O}_{2}$ and $90 \% \mathrm{~N}_{2}$, and, three times a week, counted and adjusted to $2 \times 10^{5} \mathrm{cell} / \mathrm{ml}$. At different culture times after day 7 , cells were either maintained in Mo culture condition or induced to differentiate into DCs.

\section{DC generation and maturation}

Cells recovered at different times of Mo culture or mature Mos isolated from PB were extensively washed, counted, and seeded at $2-3 \times 10^{5} / \mathrm{ml}$ in RPMI 1640 supplemented with $10 \%$ FCS and containing either IL-4 $(50 \mathrm{ng} / \mathrm{ml})$ and GM-CSF $(50 \mathrm{ng} / \mathrm{ml})$ or IL-4 $(10 \mathrm{ng} / \mathrm{ml}), \mathrm{KL}(1 \mathrm{ng} / \mathrm{ml})$ and $\mathrm{FL}$ $(1 \mathrm{ng} / \mathrm{ml})$ (Peprotech). After additional 6 days of culture, DCs were induced to terminal maturation by an overnight incubation with $1 \mu \mathrm{g} / \mathrm{ml}$ of LPS (Sigma-Aldrich). In some experiments, cells were stimulated with 3 or $5 \mu \mathrm{g} / \mathrm{ml}$ of CpG oligodeoxynucleotide. The CpG 2006 oligodeoxynucleotide, TCGTCGTTTTGTCGTTTTGTCGT ${ }^{50}$ was purchased from MWG biotech AG (Ebersberg, Germany). IFN- $\alpha$ secretion was measured by HuIFN- $\alpha$ ELISA kit (PBL Biomedical Laboratories, Piscataway, NJ USA).

\section{Immunofluorescence and morphological analyses}

Cells $\left(5 \times 10^{4}\right)$ were stained with the following FITC- or PE-conjugated MoAbs: CD1a, CD11c, CD11b, CD31, CD14, CD34, CD40, CD80, CD83,
CD86, CD123, MHC class I, MHC class II (Pharmingen, San Diego, CA, USA), anti-GM-CSFR (Serotec Ltd, Oxford, UK) BDCA-2 and BDCA-4 (Miltenyi Biotec). The appropriate conjugated isotype-matched IgGs were used as control. After incubation at $4^{\circ} \mathrm{C}$ for $30 \mathrm{~min}$ with saturating concentrations of MoAbs or isotypic controls, cells were washed twice with $\mathrm{PBS} / 0.1 \%$ bovin serum albumin (BSA) and analyzed on a FACScan (Becton-Dickinson). GFP-transduced cells $\left(5 \times 10^{4}\right)$ were washed with PBS/BSA, resuspended and fixed in $0.2 \mathrm{ml}$ of $1 \%$ paraformaldehyde in PBS (Sigma Diagnostics, St Louis, MO, USA) and analyzed by FACScan.

Cells $\left(2 \times 10^{4}\right)$ collected at different days of culture were cytocentrifuged onto glass slides, stained with May-Grünwald Giemsa, and identified by morphological analysis.

\section{Cell sorting}

The $\mathrm{CD} 34^{+} 14^{+}$and $\mathrm{CD} 34^{+} 14^{-}$cell fractions were sorted 2 days after CD34 ${ }^{+}$HPC purification. Mo cells at day 12 of culture were labeled with the appropriate MoAbs and then separated into $\mathrm{CD}^{-} 6^{-} \mathrm{GM}-\mathrm{CSFR}^{-}$, $\mathrm{CD}^{-} 6^{-} \mathrm{GM}^{-\mathrm{CSFR}}{ }^{+}, \mathrm{CD}^{+} 6^{+} \mathrm{GM}-\mathrm{CSFR}^{+}$fractions. Reanalysis of the sorted populations showed that purity was higher than $98 \%$.

\section{FITC-dextran uptake}

Cells $\left(5 \times 10^{4}\right)$ were incubated with $1 \mathrm{mg} / \mathrm{ml}$ of FITC-dextran (SigmaAldrich) for $30 \mathrm{~min}$ at either 37 or $4^{\circ} \mathrm{C}$. After several washings with cold PBS/0.1\% BSA the intensity of cell fluorescence was measured by flow cytometry. The level of antigen uptake was indicated by fluorescence difference between the test at $37^{\circ} \mathrm{C}$ and the control tubes at $4^{\circ} \mathrm{C}$ for each sample.

\section{Allogeneic MLR assay}

T lymphocytes $\left(1 \times 10^{5}\right)$ isolated from PB of healthy donors, as previously described, ${ }^{51}$ were seeded in each well of round-bottomed 96-well microtiter plates and incubated 6 days at $37^{\circ} \mathrm{C}$ in the presence of graded numbers of $16 \mathrm{~h}$ LPS-stimulated DCs or Mos. During the last $18 \mathrm{~h}$ of incubation, cells were pulsed with methyl- ${ }^{3} \mathrm{H}$ thymidine $(1 \mu \mathrm{Ci} /$ well, ICN Biomedicals, Irvine, CA, USA), harvested using a 96-well cell harvester (Tomtec, Hamden, CT, USA) and the thymidine incorporation was measured in a Microbeta counter (Wallac, Turku, Finland). Tests were performed in quadruplicate and the results are expressed as mean $\mathrm{cpm} \pm$ S.D.

\section{Production of the lentivirus containing GFP}

Lentiviral vectors were prepared by cotransfecting $3.5 \mu \mathrm{g}$ of vesicular stomatitis virus $\mathrm{G}$ protein envelope-encoding plasmid, $6.5 \mu \mathrm{g}$ of the packaging construct and $10 \mu \mathrm{g}$ of the cytomegalovirus-GFP transfer vector into human kidney 293T cells, through the LipofectAMINE 2000 kit (Invitrogen, Frederick, MD, USA) following manufacturer's recommendations. Supernatants were collected $36-40 \mathrm{~h}$ post-transfection, clarified and stored at $-80^{\circ} \mathrm{C}$ until use. Viral titers of supernatants were usually in the range of $8 \times 10^{5}-8 \times 10^{6}$ transducing units (TUs) $/ \mathrm{ml}$.

\section{Gene transduction}

Transduction of cells by lentiviral vectors was performed by adding 1-2 $\mathrm{ml}$ of viral supernatant and $4 \mu \mathrm{g} / \mathrm{ml}$ of polybrene to $3 \times 10^{5}$ cells (multiplicity of infection from 5 to 50 ) and centrifuging at $600 \times g$ for $90 \mathrm{~min}$ at $32^{\circ} \mathrm{C}$. A second cycle of infection was performed after overnight incubation in the 
appropriate culture medium. The efficiency of the infection was evaluated by flow cytometry as percentage of GFP positive cells.

\section{Acknowledgements}

We thank M Blasi, M Fontana and Dr. S Hourshid for editorial assistance and $\mathrm{G}$ Loreto and $\mathrm{A}$ Zito for graphics.

\section{References}

1. Steinman RM (1991) The dendritic cell system and its role in immunogenicity. Annu. Rev. Immunol. 9: 271-296

2. Banchereau J and Steinman RM (1998) Dendritic cells and the control of immunity. Nature 392: 245-252

3. Thery $C$ and Amigorena $S$ (2001) The cell biology of antigen presentation in dendritic cells. Curr. Opin. Immunol. 13: 45-51

4. Banchereau J, Briere F, Caux C, Davoust J, Lebecque S, Liu YJ, Pulendran B and Palucka K (2000) Immunobiology of dendritic cells. Annu. Rev. Immunol. 18: $767-811$

5. Dalloul AH, Patry C, Salamero B, Canque B, Grassi F and Schmitt C (1999) Functional and phenotype analysis of thymic $\mathrm{CD} 34^{+} \mathrm{CD} 1 \mathrm{a}^{-}$progenitor-derived dendritic cells: predominance of $\mathrm{CD}^{2} \mathrm{a}^{+}$differentiation pathway. J. Immunol. 162: 5821-5828

6. Santiago-Schwarz F (1999) Positive and negative regulation of the myeloid dendritic cell lineage. J. Leukoc. Biol. 66: 209-216

7. Galy A, Travis M, Cen D and Chen B (1995) Human T, B, natural killer, and dendritic cells arise from a common bone marrow progenitor cell subset. Immunity 3: 459-473

8. Res PCM, Couwenberg F, Vyth-Dreese FA and Spits H (1999) Expression of pT $\propto$ mRNA in a committed dendritic cell precursor in the human thymus. Blood 94: 2647-2657

9. Spits H, Couwenberg F, Bakker AQ, Weijer K and Uittenbogaart $\mathrm{CH}$ (2000) Id2 and Id 3 inhibit development of $\mathrm{CD} 34^{+}$stem cells into predendritic cell (pre-DC)2 but not into pre-DC1: evidence for a lymphoid origin of pre-DC2. J. Exp. Med. 192: $1775-1783$

10. Ardavin C, Wu L, Li C and Shortman K (1993) Thymic dendritic cells and T cells develop simultaneously in the thymus from a common precursor population. Nature 362: 761-763

11. Wu L, Li C and Shortman K (1996) Thymic dendritic cell precursors. Relationship to the $T$ lymphocyte lineage and phenotype of the dendritic cell progeny. J. Exp. Med. 184: 903-911

12. Saunders D, Lucas K, Ismaili J, Wu L, Maraskovsky E, Dunn A and Shortman K (1996) Dendritic cell development in culture from thymic precursor cells in the absence of granulocyte/macrophage colony-stimulating factor. J. Exp. Med. 184: $2185-2196$

13. Liu YJ (2001) Dendritic cell subsets and lineages, and their functions in innate and adaptive immunity. Cell 106: 259-262

14. Caux C, Vanbervliet B, Massacrier C, Dezutter-Dambuyant C, de Saint-Vis B, Jacquet C, Yoneda K, Imamura S, Schmitt D and Banchereau J (1996) CD34 $4^{+}$ hematopoietic progenitors from human cord blood differentiate along two independent dendritic cell pathways in response to GM-CSF + TNF $\alpha$. J. Exp. Med. 184: 695-706

15. Cella M, Sallusto $F$ and Lanzavecchia $A$ (1997) Origin, maturation and antigen presenting function of dendritic cells. Curr. Opin. Immunol. 9: 10-16

16. Reid CDL (1997) The dendritic cell lineage in haematopoiesis. Br. J. Haematol. 96: 217-223

17. Caux C, Massacrier C, Vanbervliet B, Dubois B, Durand I, Cella M, Lanzavecchia A and Banchereau J (1997) CD34 ${ }^{+}$hematopoietic progenitors from human cord blood differentiate along two independent dendritic cell pathways in response to granulocyte-macrophage colony-stimulating factor plus tumor necrosis factor $\alpha$. II. Functional analysis. Blood 90: $1458-1470$

18. Ogawa M (1993) Differentiation and proliferation of hematopoietic stem cells. Blood 81: 2844-2853

19. Clark SC and Kamen R (1987) The human hematopoietic colony-stimulating factors. Science 236: 1229-1237
20. Herbst B, Kohler G, Mackensen A, Veelken H, Kulmburg P, Rosenthal FM, Schaefer HE, Mertelsmann R, Fisch P and Lindemann A (1996) In vitro differentiation of $\mathrm{CD} 34^{+}$hematopoietic progenitor cells toward distinct dendritic cell subsets of the Birbeck granule and MIIC-positive Langerhans cell and the interdigitating dendritic cell type. Blood 88: 2541-2548

21. Arrighi JF, Hauser C, Chapuis B, Zubler RH and Kindler V (1999) Long-term culture of human $\mathrm{CD} 34^{+}$progenitors with FLT3-ligand, thrombopoietin, and stem cell factor induces extensive amplification of a $\mathrm{CD} 34^{-} \mathrm{CD} 14^{-}$and a CD34-CD14 dendritic cell precursor. Blood 93: 2244-2252

22. Caux C, Dezutter-Dambuyant C, Schmitt D and Banchereau J (1992) GM-CSF and TNF $\alpha$ cooperate in the generation of dendritic Langerhans cells. Nature 360: 258-261

23. Peters JH, Xu H, Ruppert J, Ostermeier D, Friedrichs D and Gieseler RK (1993) Signals required for differentiating dendritic cells from human monocytes in vitro. Adv. Exp. Med. Biol. 329: 275-280

24. Romani N, Gruner S, Brang D, Kampgen E, Lenz A, Trockenbacher B, Konwalinka G, Fritsch PO, Steinman RM and Schuler G (1994) Proliferating dendritic cell progenitors in human blood. J. Exp. Med. 180: 83-93

25. Sallusto $F$ and Lanzavecchia A (1994) Efficient presentation of soluble antigen by cultured human dendritic cells is maintained by granulocyte/macrophage colony-stimulating factor plus interleukin 4 and downregulated by tumor necrosis factor $\alpha$. J. Exp. Med. 179: 1109-1118

26. Caux C, Vanbervliet B, Massacrier C, Durand I and Banchereau J (1996) Interleukin-3 cooperates with tumor necrosis factor $\alpha$ for the development of human dendritic/Langerhans cells from cord blood $\mathrm{CD} 34^{+}$hematopoietic progenitor cells. Blood 87: 2376-2385

27. Buelens C, Bartholome EJ, Amraoui Z, Boutriaux M, Salmon I, Thielemans K, Willems F and Goldman M (2002) Interleukin-3 and interferon acooperate to induce differentiation of monocytes into dendritic cells with potent helper T-cell stimulatory properties. Blood 99: 993-998

28. Ebner S, Hofer S, Nguyen VA, Furhapter C, Herold M, Fritsch P, Heufler C and Romani N (2002) A novel role for IL-3: human monocytes cultured in the presence of IL-3 and IL-4 differentiate into dendritic cells that produce less IL-12 and shift Th cell responses toward a Th2 cytokine pattern. J. Immunol. 168: 6199-6207

29. Zou GM and Tam YK (2002) Cytokines in the generation and maturation of dendritic cells: recent advances. Eur. Cytokine Netw. 13: 186-199

30. Shurin MR, Esche C and Lotze MT (1998) FLT3: receptor and ligand. Biology and potential clinical application. Cytokine Growth Factor Rev. 9: 37-48

31. Maraskovsky E, Brasel K, Teepe M, Roux ER, Lyman SD, Shortman K and McKenna HJ (1996) Dramatic increase in the numbers of functionally mature dendritic cells in Flt3 ligand-treated mice: multiple dendritic cell subpopulations identified. J. Exp. Med. 184: 1953-1962

32. Maraskovsky E, Daro E, Roux E, Teepe M, Maliszewski CR, Hoek J, Caron D, Lebsack ME and McKenna $\mathrm{HJ}(2000)$ In vivo generation of human dendritic cell subsets by Flt3 ligand. Blood 96: 878-884

33. Smith MA, Court EL and Smith JG (2001) Stem cell factor: laboratory and clinical aspects. Blood Rev. 15: 191-197

34. Gabbianelli M, Pelosi E, Montesoro E, Valtieri M, Luchetti L, Samoggia P, Vitelli L, Barberi T, Testa U, Lyman S and Peschle C (1995) Multi-level effects of flt3 ligand on human hematopoiesis: expansion of putative stem cells and proliferation of granulomonocytic progenitors/monocytic precursors. Blood 86: 1661-1670

35. Van Snick J (1990) Interleukin-6: an overview. Annu. Rev. Immunol. 8: 253-278

36. Hirano T (1992) The biology of interleukin-6. Chem. Immunol. 51: 153-180

37. Hirano T (1998) Interleukin-6 and its receptor: ten years later. Int. Rev. Immunol. 16: 249-284

38. Ferrero E, Bondanza A, Leone BE, Manici S, Poggi A and Zocchi MR (1998) $\mathrm{CD}_{14}{ }^{+} \mathrm{CD} 34^{+}$peripheral blood mononuclear cells migrate across endothelium and give rise to immunostimulatory dendritic cells. J. Immunol. 160: 2675-2683

39. Olweus J, BitMansour A, Warnke R, Thompson PA, Carballido J, Picker LJ and Lund-Johansen F (1997) Dendritic cell ontogeny: a human dendritic cell lineage of myeolid origin. Proc. Natl. Acad. Sci. USA 94: 12551-12556

40. MacDonald KPA, Munster DJ, Clark Gj, Dzionek A, Schmitz J and Hart DNJ (2002) Characterization of human blood dendritic cell subsets. Blood 100: $4512-4520$ 
41. Strobl H, Bello-Fernandez C, Riedl E, Pickl WF, Majdic O, Lyman SD and Knapp W (1997) Flt3 ligand in cooperation with transforming growth factorbeta1 potentiates in vitro development of Langerhans type dendritic cells and allows single-cell dendritic cell cluster formation under serum-free conditions. Blood 90: 1425-1434

42. Bernstein ID, Andrews RG and Zsebo KM (1991) Recombinant human stem cell factor enhances the formation of colonies by $\mathrm{CD} 34^{+}$and $\mathrm{CD} 34+\mathrm{lin}^{-}$cells, and the generation of colony-forming cell progeny from $\mathrm{CD}_{3} 4^{+}$lin $^{-}$cells cultured with interleukin-3, granulocyte colony-stimulating factor, or granulocyte-macrophage colony stimulating factor. Blood 77: 2316-2321

43. Randolph GJ, Inaba K, Robbiani DF, Steinman RM and Muller WA (1999) Differentiation of phagocytic monocytes into lymph node dendritic cells in vivo. Immunity 11: 753-761

44. Ho CSK, Lopez JA, Vuckovic S, Pyke CM, Hockey RL and Hart DNJ (2001) Surgical and physical stress increases circulating blood dendritic cell counts independently of monocytes counts. Blood 98: 140-145

45. Chinnasamy N, Chinnasamy D, Toso JF, Lapointe R, Candotti F, Morgan RA and Hwu P (2000) Efficient gene transfer to human peripheral blood monocytederived dendritic cells using human immunodeficiency virus type 1-based lentiviral vectors. Hum. Gene. Ther. 11: 1901-1909
46. Evans JT, Cravens P, Lipsky PE and Garcia JV (2000) Differentiation and expansion of lentivirus vector-marked dendritic cells derived from human

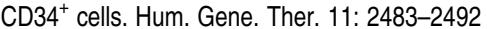

47. Oki M, Ando K, Hagihara M, Miyatake H, Shimizu T, Miyoshi H, Nakamura $Y$, Matsuzawa H, Sato T, Ueda Y, Gansuvd B, Kato S and Hotta T (2001) Efficient lentiviral tranduction of human cord blood $\mathrm{CD} 34^{+}$cells followed by their expansion and differentiation into dendritic cells. Exp. Hematol. 29: 1210-1217

48. Testa U, Fossati C, Samoggia P, Masciulli R, Mariani G, Hassan HJ, Sposi NM, Guerriero R, Rosato V, Gabbianelli M, Pelosi E, Valtieri M and Peschle C (1996) Espression of growth factor receptors in unilineage differentiation culture of purified hematopoietic progenitors. Blood 188: 3391-3406

49. Valtieri M, Gabbianelli M, Pelosi E, Bassano E, Petti S, Russo G, Testa U and Peschle C (1998) Erythropoietin alone induces erythroid burst formation by human embryonic but not adult BFU-E in unicellular serum free culture. Blood 74: $460-470$

50. Hartmann $\mathrm{G}$ and Krieg AM (2000) Mechanism and function of a newly identified CpG DNA motif in human primary B cells. J. Immunol. 164: 944-952

51. Mariotti S, Teloni R, Iona E, Fattorini L, Giannoni F, Romagnoli G, Orefici G and Nisini R (2002) Mycobacterium tuberculosis subverts the differentiation of human monocytes into dendritic cells. Eur. J. Immunol. 32: 3050-3058 